\title{
Cultural Models of Normalcy and Deviancy
}

\author{
Yulia E. Chentsova-Dutton, ${ }^{1}$ and Andrew G. Ryder ${ }^{2,3}$ \\ ${ }^{1}$ Georgetown University Washington, District of Columbia, USA \\ ${ }^{2}$ Concordia University \\ ${ }^{3}$ Jewish General Hospital, Montreal, Quebec, Canada
}

\begin{abstract}
In this article, we argue that the cultural models approach provides a useful framework for cultural-clinical psychology. We begin with a brief review of this approach before presenting the distinction between cultural models for normalcy and deviancy. As well, we consider how both normalcy and deviancy can be culturally valorised or devalorised. Given that mental disorders do not always neatly fit with available cultural models, we offer evidence-based examples of unscripted as well as scripted distress. We conclude by considering key hypothesis-generating questions and some methodological approaches that could be used to improve and extend the evidence base underpinning the cultural models approach as applied to the study of culture and mental health.
\end{abstract}

Keywords: cultural-clinical psychology, cultural models, cultural scripts, culture, distress, mental health.

People do not experience psychological distress in a cultural vacuum. Disordered emotions, cognitions, and behaviour are interpreted through conceptual lenses used by sufferers and their communities. These understandings-or cultural models - encompass what is known about particular symptoms and their combinations (models of deviancy) in reference to what is known about normal or optimal functioning (models of normalcy). Although symptoms of distress do, without a doubt, share some universal features that reflect human biology, studies also have revealed substantial cultural variation, suggesting that cultural models may not only scaffold different interpretations of symptoms and their consequences but also influence symptom experience and expression (also see Chentova-Dutton and Ryder, 2019). Past research in psychiatry has used terms such as idioms of distress or cultural concepts of distress to capture these ideas (Nichter, 2010). We rely on the language of "cultural models" (and "cultural scripts" for sequenced models), as this facilitates use of an extensive theoretical and methodological literature (Chirkov, this issue). In this article, we will demonstrate the application of a cultural models approach to mental disorder and discuss its implications for cultural-clinical psychology.

Let us begin by considering Sukhbir Anand, a

Correspondence: Yulia E. Chentsova, Georgetown University, 306 WhiteHall, 3700 O Street NW, Washington, DC 20057, USA. E: yec2@georgetown.edu or Andrew G. Ryder, Concordia University, 7141 Sherbrooke Street W (PY139-3), Montreal, Quebec H4B 1R6, Canada. E: andrew.ryder@concordia.ca 31-year-old man, originally from the Punjab but living in the Midwestern United States for almost 10 years:

Sukhbir Anand lives with his parents and is currently working at their family-owned hardware store. He suffers from sleeplessness and irritability, combined with a sense of lassitude, aimlessness, and diminished enjoyment. Moreover, his irritability in particular is making it more difficult for him to work, at times even affecting interactions with patrons at the store. Over time, these issues have placed a great strain on his relationship with his parents. Mr. Anand is referred to a White American woman from the Northeast, an experienced psychotherapist in her mid-50s. When she asks Mr. Anand on intake about his main concern, he responds that his parents and some people in his community are concerned that he has gotten so irritable. He adds that they say he is not showing proper respect and that he would do so if he had more direction in life.

Does Mr. Anand consider his symptoms to be problematic? If so, does he think they are bothersome, but normal, or indicative of some form of illness? If the latter, what does he think might happen if the illness is left untreated, and what sort of treatment might he consider?

The therapist waits several sessions before asking him again about his main concern, this time emphasising that she wants to hear about his own understanding of the problem. He replies that his main concern is that he is not yet married and that his symptoms might make find- 
ing a suitable partner more difficult, adding that this concern might be why he is struggling in the first place. The clinician takes note of both the client's and the parents' explanations, but also wonders whether family enmeshment might be playing a role. She considers whether she should directly question Mr. Anand about why he still lives at home and whether his desire for marriage is really his own goal or that of his parents.

Mr. Anand's understanding of his symptoms cannot be considered in isolation from his local social world; thus, a number of questions arise from his situation. Which models of normal behaviour are being violated here such that the distress is identified as pathological?

What does the larger community think about these kinds of problems? How might these understandings actually feed into his illness experience? What should he-and his parents, his community, his therapist-do? Cultural psychiatry and medical anthropology have grappled with these kinds of questions for several decades, joined in recent years by cultural-clinical psychology (Ryder et al., 2011). Although there is broad consensus on the importance of understanding both individually and consensually held beliefs about mental disorder and their implications, the field lacks a framework for doing so. Our overarching goal in this article is to demonstrate that a cultural models approach, grounded in cognitive science, can provide such a frame-work, contributing a common language, a way of thinking, and a set of methods that would benefit researchers and clinicians. We begin with an overview of the approach, first considering models of normalcy that constitute the backdrop against which psychological deviancy is understood before turning to models of deviancy themselves. We also explore the distinction between unscripted and scripted distress. We conclude by discussing a number of research questions and methodologies which could be used to address these questions.

\section{Models and Scripts}

"The systematic neglect of culture is the single biggest barrier to attaining the highest available standard of health worldwide"-so argued the 27 authors of a commissioned review for The Lancet (Napier et al., 2014). Scholars across disciplines are dedicated to addressing this neglect. Over the past decade, cultural-clinical psychology has begun the work of integrating cultural and clinical approaches while drawing on a long research tradition in psychology that stretches back to the 1970s (Draguns, 1980; Marsella \& Yamada, 2007; Tanaka-Matsumi \& Draguns, 1997). The resulting subdiscipline is, moreover, increasingly engaged in the interdisciplinary conversation of cultural psychiatry, which itself has a rich literature stretching back for decades (e.g., see reviews of "the new cross-cultural psychiatry:" Kirmayer, 2006; Kleinman, 1977; Littlewood, 1990). Both traditions have shared progression from cataloguing group similarities and differences to examining mechanisms that account for these differences. There is also a long-standing and shared interest in the normative models of psychological processes that form the background against which mental disorder is understood. The time is ripe for integration: Cultural and clinical psychologists alike can benefit from seeing the phenomena in which they are interested through the lens of cultural models of what is and is not considered normal.

\section{The Cultural Models Approach}

We begin with the cultural models approach developed within cognitive anthropology, a field that-like cultural psychology - is concerned with both cultural contexts and individual minds, as well as their mutual influences (d'Andrade, 1995)

Cultural models are presupposed, taken-for-granted models of the world that are widely shared (although not necessarily to the exclusion of other, alternative models) by the members of a society and that play an enormous role in their understanding of that world and their behavior in it. (Quinn \& Holland, 1987, p. 4)

Cultural models are understood to be "in the head" as beliefs, internalised norms, and values as well as "in the world" as observable behaviour and cultural products (also referred to as "conventional" models; Shore, 1998, p. 46). For example, in the domain of mental disorders, cultural models exist as folk beliefs (and professional beliefs shared by practitioners) as well as depictions that appear in stories or media. Models that are "in the head" typically include aspects of knowledge that reflect broadly consensual internalised versions of shared models, and beliefs that are informed by idiosyncratic episodic memories and affective responses (Abelson, 1981). Shore (1998, p. 46-47) made a similar distinction between "conventional mental models" and "personal mental models", respectively. A person's representation of mental illness may reflect some version of culturally consensual beliefs, such as diagnostic criteria for major depression or consensual ideas about "nerves," but may also be shaped by personal experiences, such as supporting a friend through an episode of anxiety.

As cultural researchers, we are primarily interested in consensual knowledge shared by the public, but as clinicians, we are also concerned with internalised and idiosyncratic beliefs as they may inform a given person' thoughts about their own experience. Throughout this article, we will focus on the widespread cultural models held and reproduced by the public. Traditional healing and contemporary biomedicine can also be understood as subcultural systems in themselves (Kleinman, 1974, 1978); "expert” cultural models shared by 
clinicians will not be emphasised here for lack of space (for an excellent examination of this topic, see Luhrmann, 2000).

Cultural models are often contested within a given cultural context (e.g., alternative ways to conceptualise depression and anxiety in the United States: Nations et al., 1988), with additional heterogeneity generated by subcultures (e.g., sets of alternative models of problemas de nervos shared by Portuguese immigrants to North America: James et al., 2009). Dominant cultural models are nonetheless broadly shared, in two senses: First, many people in a given local social world come to hold broadly similar beliefs; second, they come to assume that others in this social world are operating under similar assumptions. Even people who contest the dominant model share knowledge about what is being contested. This common ground of intersubjectivity may at times play a greater role in predicting behaviour than do a person's own beliefs (Chiu et al., 2010; Gelfand \& Harrington, 2015). Cultural models shape the planning and execution of actions, based in part on the assumption that other people will interpret these actions in a culturally meaningful way (Kronenfeld, 2008; Quinn, 2011). For the researcher and the clinician alike, a cultural models approach requires one to begin with idiosyncratic and locally shared aspects of the models while looking ahead to within-culture and cross-cultural comparisons (Chirkov, 2016; Wassmann et al., 2011).

Interest in cultural models began with anthropologists in the 1950 s and 1960s, growing rapidly during the cognitive revolution and the ensuing explosion of interest in how knowledge was learned, stored, categorised, and recalled (Quinn, 2011). One consequence, however, was a parallel proliferation of terminology with different researchers using terms such as schema, script, frame, scene, or scenario, in addition to model, each with their own nuances (Bateson, 1972; Keller, 1992; Minsky, 1975; Rumelhart, 1980; Schank $\&$ Abelson, 1977). We will use the term model here generically to cover this family of terms, especially when describing complex constellations of schemas and scripts (e.g., "a cultural model of public interactions"). In our view, cultural models include (a) the shared subjectivity that comes from people in a given local social world having access to broadly similar beliefs; (b) observable correlates of these beliefs, such as behaviour or depictions in cultural products; and (c) the intersubjectivity that comes from believing that these beliefs are widely shared within the social world and understanding that others may interpret observable behaviour or cultural representations in a manner similar to one's own (and indeed, that these metabeliefs are themselves widely shared).

We will shift to the term cultural script to describe more specific aspects of broader models that are encoded as cognitive or behavioural sequences (e.g., "a script of grieving"). Dating back to Schank and Abelson (1977), "script" emphasises observable sequences of actions as well as understand- ing of these sequences. Over time, the term expanded to mental processes that are understood as sequenced. Many, although by no means all, cultural models of mental illness are script-like. Temporality is important for models of mental illness, as it is in keeping with the onset and course of many mental disorders or history of seeking treatment. Unlike the official diagnostic categories, the informal categories used by professionals and lay people alike are script-like (Kim \& Ahn, 2002a, 2002b). A continuum can be described with classical, rigidly filled-in scripts at one end and looser sketchy scripts at the other (Reisbeck, 1985; Scholtens, 1991). We will largely emphasise scripts toward the latter end of that continuum.

\section{Functions of Cultural Models}

Humans are faced with the ever-present problem of complexity management (Hirsh et al., 2012). At any given moment, our phenomenological fields encompass a vast array of changes that can potentially capture our attention and shape possibilities for action. Most forms of distress emerge as even more confusing sets of potential symptoms, puzzles to be solved by sufferers, those close to them, and those treating them. Human perception and cognition make use of cultural models to foster prototype-based processing of complex and confusing noncategorical phenomena.

As an example, consider the way we think about personality types, facial expressions, or color categories (see Harnad, 2003). In all of these domains, the underlying information, such as sensory input or behavioural observations, is dimensional and complex. There are no sharp boundaries between behaviour of the introverts and extraverts or the colors green and blue. The fuzzy categorical boundaries we perceive so effortlessly are due to our exposure to and experience with culturally entrained prototypes (Rosch, 1973; e.g., what blue looks like) and models (e.g., models of emotion or personality). Some of these concepts are script-like in nature, helping us perceive sequences (e.g., emotions: Siemer, 2008). There is variation in how these conceptual spaces can be carved up in different cultural and linguistic contexts, including whether folk concepts are organised as conceptual categories versus dimensions. Studies uncover cultural group differences at the level of specific categories (e.g., language-dependent color categories) along with similarities in the underlying dimensions (e.g., wavelength and brightness influencing color perception; see Regier and Kay, 2009). The same logic can be used to describe how we extract normative ideas such as "a polite person" or "justifiable anger"—and, as we shall see, concepts of deviance such as "burnout" or "problem drinking." In these domains as well, categorical prototypes guide our perception of complex stimuli.

Recruitment of models helps us function in our cultural worlds, allowing us to not continually figure out what is happening, where to attend, or how to act. A patron visiting the 
hardware store owned by Mr. Anand's parents is likely to follow a cultural script grounded in this model; arriving at the store, looking for what is needed, saying "Excuse me" before asking for help, and making minimal small talk while paying the sticker price. There is a particular sequence that gets us from the front door to the needed item and then to the cashier; this sequence is known to the patron, other patrons in the store, and people working in the store. It is publicly observable as behaviour and encoded in minds. In another cultural context, the same type of interaction might require greeting the owner upon arriving, drinking tea, getting in line, or haggling over the price. Importantly, different cultural scripts for visiting a hardware store are not isolated behavioural recipes, as they draw on other models (norms of social interactions, emotions that should or should not be expressed, personal boundaries, beliefs about social hierarchy). Specific scripts and broader models that they engage allow us to spend our limited cognitive resources on finding what we need, planning dinner, and so on, rather than figuring out how to talk to the clerk. Under normal circumstances, there is little need for people to reflect on the model. The specific content fades into the background of our consciousness as part of the shared context that ensures we do not engage in isolated behaviours but rather meaningful acts (Bruner, 1990; Searle, 1980). Cultural models make this possible.

Because a shared local social world provides many opportunities to encounter the theme through its many variations, personal models are likely to include elements that are broadly similar as well as those that differ person-to-person (Chirkov, 2016). As described earlier, the shared, consensual nature of these models does not preclude individual experience. Each person has a unique learning history and resulting access to ideas, and this history is reflected in their personal models, which, as described earlier, include aspects of internalised shared models as well as idiosyncratic elements and may therefore vary from models consensually held in the local social world (Jones et al., 2011; Shore, 1998).One patron in a store may have a complicated question about a hard-to-find tool, another one might know the family; we might then observe longer interactions than are typical, the first more detailed and technical, the second one warmer and more personal. People can also vary in their exposure to the prevailing cultural model (Kronenfeld, 2011); here we see an older person who maintains a somewhat out-dated formality, and there we see an immigrant who tests whether the store owners are willing to haggle. Complicating matters further, a given person's internalised version of the model may be incomplete. Although a socially anxious person may have access to the shared cultural model of how one shops, some aspects of the model (e.g., how one asks for a correct price when noticing an error on the receipt) may be underdeveloped as a result of social anxiety, and others may include idiosyncratic elements (e.g., an experience of a panic attack in response to being approached by another customer during a previous visit might impact beliefs about going to the store).

Personal models have social functions even when they are idiosyncratic. They are discussed with others, performed for others, and used to understand others. The essential intersubjective element means that guide our best guesses about what others are thinking and why they are acting the way they are acting - and our best guesses about how others will think about what we are thinking, and how they will interpret our actions, grounding our communication (Clark \& Brennan, 1991). In a given interaction, it is not necessarily the case that everyone is drawing on the same cultural model in the same way, but successful communication will depend on mutually adjusting (Bennardo \& Kronenfeld, 2011).). Dressler (2004, 2012, 2017) demonstrated that cultural consonance, being able to live in accord with highly valued cultural models, is associated with positive health outcomes. In sum, cultural models help us navigate the physical and social world. We learn them through social life in a particular community of minds, each with its own set of common experiences and available knowledge, along with a particular set of observable behaviours and cultural products shared with others. Once acquired, cultural models aid us in making our experience meaningful. Distress is a challenge to meaning, and not only through failures of cultural consonance. The very fact that one is distressed may in some cases violate a cultural model (e.g., the pursuit of happiness, the need for stoicism). Moreover, culturally unusual presentations of distress may break the bounds of our available models. It is to these circumstances, and how we might best understand them within the cultural models framework, that we now turn.

\section{From Normalcy to Deviancy}

Until this point, we have emphasised models that are widespread, guiding normative interpretations and every-day behaviours. What about models of nonnormative psychological experiences? Our focus for the remainder of this article is on what we refer to as cultural models and scripts for deviancy and the ways in which these models can be better understood-and studied - through closer ties to the literature on normative cultural models. When changes associated with distress are first noticed, people recruit explanations available in their cultural contexts, based on how these changes relate to their understanding of normality as well as to known kinds of problems.

We thus argue that researchers studying distress should maintain a dual focus on cultural models of normality and deviancy. One challenge here is that these sets of models have been studied within different literatures. Whereas normative cultural models (e.g., for behaviour, self-regulation, mental, bodily, social, and spiritual functioning) are the province of cultural psychology, cognitive anthropology, cultural/cognitive sociology, or semiotics, models of mental ill- 
ness are described and studied by clinical psychology and psychiatry. In the latter, there has either been little attention to culture or reliance on an assumption that adherence to "Western" models is a sign of "mental health literacy" (for a critique see Na et al., 2016).

What little crossover exists can be found in hybrid disciplines such as cultural psychiatry or cultural-clinical psychology (Bhui \& Bhugra, 2002; Karasz, 2005; Kirmayer, 2005; Ryder et al., 2011; Weiss, 1997). This article aims to connect these literatures, examining ways in which models of normalcy and deviancy inform each other.

A dual focus on normalcy and deviancy is much more useful to the clinician than is merely counting symptoms. Mr. Anand might feel irritated because he has experienced difficulty pursuing and attaining culturally valued goals; his irritability might be identified as pathological because it violates a normative cultural model for social behaviour and emotional expression (e.g., polite behaviour with strangers); indeed, he might pathologise his own irritability precisely because he can detect from the reactions of others that he is violating their expectations (Giosan et al., 2001; Thoits, 1985). Although he may be struggling to make sense of his experience through prevailing normative models, he may come to understand what is happening by means of cultural scripts for deviancy (e.g., nervous breakdown, "sinking heart, " a Punjabi idiom of distress, "intermittent explosive disorder"), as may his family, friends, and any health professionals. In turn, these cultural scripts may play an important role in further shaping his illness experience and the expression of his symptoms. Consider the family of cultural scripts for presenting emotional distress to a health professional that might be shaping Mr. Anand's discussion with his psychotherapist. The script of what to share might be linked to models and scripts for the unfolding of particular symptoms such as worry, fatigue, or anger outbursts.

A visualisation of the relation between commonly shared cultural models and scripts for normalcy and deviancy is presented in Figure 1. People in need of clinical care, their loved ones, or community members in our research studies may be relying on these sets of models when reasoning about mental health and mental illness. Vertically, we see the distinction between normalcy and deviancy; horizontally, we move from models and scripts that are culturally valorised (i.e., valued, described as optimal) to those that are culturally devalorised (i.e., pathologised, described as suboptimal or problematic), with a middle column for those that are culturally unmarked. Note that although most common forms of mental illness are dimensional (Kotov et al., 2017), with the key dimensions replicating across cultures (Carragher et al., 2015; Krueger et al., 2003), folk models of mental illness tend to combine prototype-based categories and dimensions (see Haslam, 2005; Schoeneman et al., 1993). People commonly perceive essentialised kinds or syndromes, as informed by cultural mod- els that describe them as such (Prentice \& Miller, 2007). It is an empirical question whether normalcy and deviancy are represented as prototype-based categories or dimensions in a given cultural context. For the sake of simplicity, we will describe them as categories here.

Although cultural scripts for normalcy do not generally reflect the presenting complaints of clients, they provide the frames by which these complaints are understood by the clinician and by others in the sufferer's social world. Valorised models of normalcy represent the "cultural target," a prototype that is often difficult to achieve but provides a standard for comparison (e.g., a shared script about a "go-getter:" someone persistent who works hard to pursue goals). Then we have unmarked models and scripts for normalcy, representing what is understood as "normal" in a given milieu (e.g., an "ordinary Joe," whose motivation fluctuates). Of course, normalcy is not limited to that which receives widespread approval or even acceptance in a given cultural context. Normal variations in psychological functioning might cause difficulties and problems in living without being understood as deviancy or needing an illness label (Kirmayer, 2005). Culturally devalorised models and scripts are not marked out as deviant or pathological in any way, but are consensually understood as suboptimal characteristics (e.g., "a lazy person" that is not motivated to work). Although such problems are not ascribed pathologised status, Haslam (2016) described the phenomenon of "concept creep" in which suboptimal-but-normal variants are increasingly understood as pathological, and in need of a pathologising label, over time (i.e., shifting from normalcy to deviancy in our figure). Cultural contexts also vary in terms of how much deviation from the norm is tolerated (Gelfand et al., 2011). Moreover, subcultural groups (e.g., urban vs. rural, different ethnic groups or professional cultures) may differ when it comes to perceptions of deviations from the norms. As such, the boundary between normalcy and deviancy may shift over time or differ by context.

Shifting to models of deviancy, we can still differentiate models that are valorised, unmarked, and devalorised. On the lower row of Figure 1, culturally valorised models of deviancy represent an unusual, but important, set of models, where a person's experience is clearly problematic but is also in some way respected or even celebrated in the person's cultural context (e.g., someone who has periods of mania that boost their self-confidence and willingness to take on new projects). This script for deviance must be understood in relation to the parallel script for normalcy: The person's problem is celebrated because it fits well with the "go-getter" script, even as it might cause problems in other domains. Unmarked models or scripts for deviancy are clearly pathological, but not especially celebrated or condemned beyond the general devaluing due to reduced functioning (e.g., insomnia and low mood after witnessing a car accident). Culturally 


\section{Figure 1}

A framework for cultural models and scripts: normalcy-deviancy and valorised-devalorised.

\begin{tabular}{|c|c|c|c|}
\hline \multirow{2}{*}{ Normalcy } & Valorized & Unmarked & Devalorized \\
\hline Deviancy & Ideal & Typical & Problematic \\
\hline $\begin{array}{c}\text { Pathologised (but also } \\
\text { respected/celebrated) }\end{array}$ & Pathologised & Pathologised (and stigmatised) \\
\hline
\end{tabular}

devalorised models of deviancy add stigmatising negative judgements, both self-and socially imposed (e.g., depressed mood due to "workplace burn-out" understood as weakness, laziness, or even malingering). Finally, outside the figure entirely lies unscripted distress, where the suffering defies available categories (e.g., extremely vivid dreams and extensive sleepwalking in a cultural context that does not provide a framework for explaining such an experience). Note that we are referring to broadly shared models. There may be an available, albeit obscure, diagnostic label and perhaps even consensus among clinicians (e.g., a sleep disorder) — but no popular awareness, let alone consensus.

Having briefly outlined these types of models, let us further explore the implications of experiencing psychological symptoms that match or fail to match cultural models of deviancy. How would it affect the ability of a sufferer, like Mr. Anand, to detect problems? What about people close to Mr. Anand? And how might sufferers and close others respond to these problems once detected?

\section{Unscripted and Scripted Experiences of Deviancy}

Personal signatures of distress and cultural models and scripts of how psychological functioning can go awry are not reducible to each other: Mr. Anand's unique experience of irritability is not identical to shared Punjabi or North American models of how irritability affects a person or scripts of how different forms of treatment affect irritability. His experience may not even match his pre-existing personal models of this domain; indeed, discrepancies between symptoms of a given individual and available scripts of deviancy are important. Consider the sociological research on Russians who are active on acquired immune deficiency syndrome (AIDS)-denialist online communities. Some of the newcomers to these communities are people trying to make sense of their human immunodeficieny virus (HIV)-positive status in light of the fact that their individual symptoms do not match the dominant script for HIV and AIDS (Meylakhs et al., 2014). The discrepancies sow doubt and trigger the search for alternative scripts, such as those promoted by AIDS-denialists.

Neither are personal experiences and cultural models independent; the former are framed by the latter, and the latter are maintained or changed in part by the former (Kirmayer, 2005). When the sufferer's changes in functioning match cultural models for deviancy that are available in a given context, symptoms can be described as "scripted distress." The changes can be understood and described to others using known terms, with known implications. In some cases, all signs may point to a single widely shared explanation, whether due to a distinctive presentation or a dominant cultural model; in others, a number of viable models may be available, with different implications for perceived cause, potential impact, or treatment. Finally, some changes may not be understood as a coherent category, something we refer to as "unscripted deviancy."

\section{Unscripted Experiences of Deviancy}

Unscripted deviancy is characterised by confusion. Although individual symptoms may be recognised, the patterns make no apparent sense within a given cultural context. Some examples come to us from work on the history of mental illness. For example, symptoms of dissociative identity disorder, formerly known as multiple personality disorder, were not widely recognised by the lay public in the United States prior to the publication of the Three Faces of Eve and the subsequent release of the film with the same name (Johnson, 1957; Thigpen \& Cleckley, 1954, 1957). Memory lapses and abrupt changes in personality, whatever their causes, did not map onto the existing publicly shared models of physical or mental illness at that time. Clinical professionals already had begun developing a model of this form of distress by then, but it was still contested and recruited only rarely. Once the idea of this disorder entered the marketplace of ideas and propagated, a widely shared script emerged, becoming more elaborated and linked to salient cultural concerns over time. A striking example of the latter is the addition of childhood 
abuse to the script after the book and film "Sybil" (Petrie, 1976; Schreiber, 1973; see Hacking, 1992) Other examples include early reports of emerging disorder concepts such as cases of high-functioning autism spectrum disorder (Molloy \& Vasil, 2004) or chronic fatigue syndrome and fibromyalgia (1995 Yunus et al., 1981). It is not atypical for symptoms of a disorder to be scripted in one context, but not in others. Sets of changes that map onto particular models of deviancy in "non-Western" cultural contexts are sometimes unscripted in the West. Historical and modern descriptions of koro provide good examples of both clinicians and sufferers lacking explanations for subjective distressing perceptions of shrinking genitals (Crozier, 2011).

Similarly, disorders that are scripted in the West may not be scripted elsewhere. Symptoms of attention deficit hyperactivity disorder are recognised as such by larger proportions of samples in American or British cultural contexts than by those in other locales such as Korea, Malaysia, and Brazil (Gomes et al., 2007; Loo et al., 2012; Park et al., 2018; Pescosolido et al., 2008). How do people account for these symptoms when they emerge in non-Western contexts? Are there alternative models that account for them? In some of these contexts, such as Brazil, people tend to interpret such symptoms as culturally devalorised normalcy (developmental problems stemming from poor parenting and lack of discipline, Gomes et al., 2007); in others, they appear to be unscripted problems in search of viable explanations. One study was conducted with Cuban American mothers of children who exhibited attention deficit hyperactivity disorder features of inattention and impulsivity. The cultural models available to these mothers and internalised by them did not account for their children's behaviour, leading to confusion and an active search for explanations (Arcia \& Fernandez, 1998). The article cites one of the mothers as saying: "What could he possibly have? What could it possibly be?" (p. 339), highlighting angst at encountering culturally unscripted symptoms. The authors argued that Cuban cultural models of normal child development focus on other, more culturally relevant, indicators of functioning, such as intelligence and warmth, with no explanation for impairing symptoms that nonetheless leave intelligence and warmth intact.

These reports make it clear that people find experiences of unscripted distress to be challenging over and above the negative effects of the symptoms themselves. A large body of research has suggested that experiences that violate our expectations and undermine our sense of meaning are unsettling, triggering anxious arousal (Proulx \& Inzlicht, 2012). People reporting unscripted symptoms become anxious and confused and doubt their own perceptions (Arcia \& Fernandez, 1998; Woodward et al., 1995). Social support and treatment are harder to recruit for problems that are difficult to describe, or even to believe (Dumit, 2006; Ware, 1992).

Given this, people who suffer from unscripted symptoms tend to actively search and advocate for potential explanations, whether they are or are not accurate. Although reactions vary and the possibility for stigma increases for some types of symptoms, people have commonly reported that gaining access to potential cultural model(s) for one's symptoms via receipt of diagnostic labels is perceived as helpful (Molloy \& Vasil, 2004; Wisdom \& Green, 2004; Woodward et al., 1995). For example, teens and adults with autism spectrum disorder have reported that it was comforting for them to be provided with a diagnostic category, allowing them to make sense of and reattribute their symptoms, describe them to others, and access support and treatment (Molloy \& Vasil, 2004; Punshon, Skirrow, \& Murphy, 2009). Given the thirst of sufferers for cultural models, it is not surprising that new models and scripts emerge and propagate as a result of word-of-mouth or digitally mediated communication, with social media fueling rapid spread (Cline \& Haynes, 2001). In some cases, efforts to help others obtain information backfire, as evidenced by cases of mass psychogenic illness fueled by novel cultural models of deviancy gone awry (Bartholomew et al., 2012).

\section{Scripted Experiences of Deviancy}

When distress is scripted in a cultural context, an individual person's signature of distress maps, even if imperfectly, onto cultural models of deviancy. This personal signature is understood as a potential problem (or, more typically, a set of alternative problems) with a name and a known presentation, cause(s), course, and treatment approach. Some of these known problems may be valorised, and others devalorised.

Culturally valorised experiences of deviancy. Although all forms of mental illness interfere with cultural norms of functioning at high levels of severity, culturally valorised models of deviancy can fulfil these norms at lower levels of severity and be valued even at higher levels of severity. For example, religious obsessions and compulsions in religious people in Turkey and Israel (Karadaĝ et al., 2006; Yorulmaz \& Iş1k, 2011; Zohar et al., 2005), dissociation in the Afro-Brazilian religion of Candomble (Seligman, 2005), social anxiety in Japan (Asnaani et al., 2010), shyness in rural Chinese schools (Chen et al., 2011), excessive dieting in Medieval Europe, contemporary Western cultural contexts, and, increasingly, East Asia (Chisuwa \& O'Dea, 2010; Stice, 2001; Twamley \& Davis, 1999; Witztum et al., 2008), or narcissistic traits and hypomanic states in the United States (Ford et al., 2015; Foster et al., 2003; Martin, 2009) are relevant to and at least somewhat compatible with culturally normative and desirable spiritual pursuits, beliefs about the self, interpersonal concerns, bodily shape and fitness, or emotional states.

In these cases, cultural models provide nonpathological attributions of the symptoms (e.g., interpretation of dissocia- 
tive states as spirit possession among Candomble mediums; Seligman, 2005). Symptoms may be perceived as desirable, at least until their severity makes it clear that something has gone wrong. Martin (2009) described public perceptions of mania as having "market value" (p. 218) in the United States, even generating envy of high productivity, excitement, and energy associated with its symptoms. Similarly, shy children are perceived positively in rural Chinese schools, as demonstrated by Chen et al. (2011) and by Chen et al. (2009).

Indeed, research has suggested that on one hand, cultural factors may reinforce such symptoms or make them easier to disclose, but on the other hand make them harder to detect as problematic at lower levels of severity (Cafri et al., 2005; Heinrichs et al., 2006; Yorulmaz \& Işık, 2011). For example, one cross-cultural study has examined college students' endorsement of social anxiety symptoms and perceived cultural norms regarding withdrawn social behaviour (Heinrichs et al., 2006). The two were positively associated with each other, with higher endorsement of social anxiety symptoms in countries with higher perceived acceptance of these symptoms. As cultural models of normalcy change, so does expression of culturally compatible symptoms. Consider the case of China where rising individualistic values are associated with rising levels of narcissism (Cai et al., 2012), driving changes in what is considered normal or deviant self-regard. In sum, relatively low discrepancy between one's symptoms and cultural models of normalcy may reduce stigma, but also may raise thresholds for detecting distress or reluctance on the part of sufferers to treat relatively their culturally desirable, or perhaps even admired, symptoms.

Culturally devalorised experiences of deviancy. In contrast to valorised models of deviancy, culturally devalorised models of deviancy can be interpreted as especially troubling violations of cultural models for normalcy. Consider psychopathy in modesty-reinforcing Scottish cultural contexts (Cooke \& Michie, 1999; Cooke et al., 2005); alcohol abuse and dependence in alcohol-prohibiting Muslim-majority cultural contexts (Michalak \& Trocki, 2006); dissociation in European American cultural contexts, where it is understood as a "violation of normative selfhood" (Seligman \& Kirmayer, 2008, p. 21); posttraumatic distress in Nepal, where misfortune is often interpreted as evidence of karma due to past-life transgressions (Kohrt \& Hruschka, 2010); or anxiety and depression in war veterans with posttraumatic stress disorder in cultural contexts with strong masculine norms (Garcia et al., 2011). In these cases, symptoms signal a troubling violation of how things should be in a given cultural context, often implicating models of what it means to be a worthy person.

What are the intrapersonal and interpersonal consequences of norm-violating and devalorised forms of psychological distress? Higher levels of the underlying vul- nerability trait (e.g., impulsivity) may be required for behavioural expression. For example, in modesty-promoting Scotland, higher levels of psychopathic personality traits may be required before specific instances of crime are observed (Cooke and Michie, 1999; Walsh, 2013; but also see Wernke and Huss, 2008). The thresholds for detecting and reacting to symptoms may be lower, potentially facilitating recognition and intervention at lower levels of severity. For example, Daley (2004) observed that although Indian parents of children with autism detected and sought treatment for their children's symptoms much later than did their Western counterparts, they tended to detect social symptoms first and become concerned about them. This is unlike parents in Western studies who tend to first become alarmed by language delays. Researchers have speculated that the pattern observed in India may be due to the possibility that children's aloofness and lack of reciprocity violate culturally normative models of closeness between young children and their caretakers. The same study has suggested that Indian parents were less likely to detect symptoms perceived as consistent with cultural norms (e.g., being quiet).

Symptoms that violate cultural models of normalcy can be particularly distressing (e.g., sexual orientation obsessions in a predominantly U.S.-based sample; Williams et al., 2015) and may be perceived more negatively by others. For example, Liu et al. (2014) argued that in contrast to shyness, a trait that has traditionally been perceived positively due to its compatibility with cultural norms of modesty (and continues to be so in rural areas in China), unsociability, or lack of interest in others, violates Chinese cultural norms of relational interdependence. Even Chinese kindergarteners and first graders are aware of the cultural model linking unsociability with negative outcomes (Ding et al., 2015). Indeed, unsociability is associated with higher negative self-perceptions, emotional distress, lower academic performance, and lower peer acceptance among Chinese children and early teens (Liu et al., 2014). Unsociability is more predictive of these outcomes in China than in Canada, a cultural context that places relatively less emphasis on social connectedness (Liu et al., 2015).

When a person believes that others understand their symptoms as socially or morally inappropriate, he or she may mask them, whether consciously or not, inhibiting expression of such symptoms. Consider a study suggesting that members of a predominantly Italian American community in Pennsylvania in the 1960s were more reluctant to acknowledge and seek treatment for symptoms of mental illness as compared to their counterparts in neighbouring communities with populations of predominantly West European descent (Bruhn et al., 1966). The authors had argued that symptoms of mental illness violated Italian cultural norms more so than did the norms of neighbouring communities. Indeed, decades of research on the stigma of mental illness have suggested 
that people from different cultural contexts differ in their levels of endorsed stigma, with higher stigma for symptoms violating cultural norms (Abdullah \& Brown, 2011). In sum, despite the fact that devalorised symptoms may be detected earlier, triggering help-seeking and assumption of the sick role (Seligman \& Kirmayer, 2008), they may not be immediately expressed and addressed because it may be difficult for people to acknowledge and discuss problems that are known to be perceived negatively inter-subjectively.

Culturally unmarked experiences of deviancy. Finally, let us consider culturally unmarked scripted symptoms. Although distress or impairment caused by such symptoms would potentially interfere with local norms, the symptoms themselves would not be considered directly relevant to cultural norms. For example, although a biomedical model of panic disorder (PD) is recognised and endorsed by a substantial number of adults in the United States (Coles \& Coleman, 2010), the symptoms of PD do not map onto cultural models for normalcy in an obvious way, beyond the general distress and impairment. In such cases, cultural models for deviancy can still guide a person's beliefs about causes, consequences, and interventions.

In sum, cultural models for deviancy frame individual experiences of distress, potentially affecting detection of, conceptualisation of, and responses to symptoms. We have argued that they do not exist in isolation from cultural models of normalcy. Examining the relationship between individual signatures of distress and the ways in which one is understood to, on one hand, flourish or function normally, and on the other hand, to suffer, is critical to the effort of developing nuanced models of cultural shaping of distress, whether for research or treatment.

\section{Cultural Models of Deviancy: A Research Agenda}

The framework proposed here is meant to aid researchers in understanding ways in which cultural models of normalcy and deviancy affect mental disorders. This framework can be helpful for understanding individual experiences of distress as well as local meanings, facilitating cross-cultural research. In this section, we will consider the kinds of questions one may ask based on this framework and the types of research tools one may use to address them.

\section{What Are the Relevant Local Models or Scripts?}

When working with an individual or a sample from an understudied cultural context, researchers should start with the very basic question of whether a particular domain of distress is scripted in a local context. Imagine that Mr. Anand's psychotherapist has a colleague, a researcher who consults on this case, notes the lack of research and decides to conduct research on anger and irritability in this cultural context. As the research literature is scant, what should she do to better understand shared "in the head" and "in the world" models of this domain in Mr. Anand's context and the ways in which his personal model is similar to or different from them? She should ideally start by harvesting local terms and ideas about this domain. Then, armed with this knowledge, she could move to the private aspects of these models as experienced and reported by community members and also by any sufferers who can be identified.

Ethnography. In cases where little is known about a particular domain, we recommend starting with an ethnography. This approach deserves careful consideration, especially when entering a new cultural context (Chirkov, 2016). Infrequently used and poorly understood by psychologists, ethnographic and observational approaches are mainstays of cultural anthropology and cultural psychiatry. Even those anthropologists who advocate for procedures that include quantitative approaches have strongly underscored the importance of beginning with careful observation (Bennardo \& Munck, 2014). Although classical ethnographies often take years, with ethnographers avoiding specific research questions until deep into the project, more focussed methods have been proposed when the general question (e.g., experience of emotional distress in a given context) and research site (e.g., an outpatient unit at a psychiatric hospital in that context) are already known (Cruz \& Higginbottom, 2013; Rashid et al., 2015; Wall, 2014). In particular, a person-centered ethnographic approach - emphasising what it is like to be that kind of person, in that kind of setting - holds promise for the study of cultural models (Hollan, 1997; LeVine, 1982).

Structured and semistructured interviews. Moving beyond ethnography to more structured data collection, a researcher can probe models of mental illness with semistructured or structured interviews. A good example of a study that has documented a script for complex posttraumatic stress disorder is provided by Stadtmann et al. (2018). A number of interviews that can be used to identify and describe models and scripts of mental illness have been developed: Examples include the Barts Explanatory Model Interview (Rüdell et al., 2009), the Clinical Ethnographic Interview (Saint Arnault \& Shimabukuro, 2012), the Explanatory Models Interview Catalogue (Weiss, 1997), the McGill Illness Narrative Interview (Groleau et al., 2006), and the Short Explanatory Models Interview (Lloyd et al., 1998). The general approach has been streamlined for clinical use, with the recent inclusion of the Cultural Formulation Interview (CFI; Lewis-Ferńandez et al., 2013) in the Diagnostic and Statistical Manuel of Mental Disorders, fifth edition (American Psychiatric Association, 2013). Although the CFI includes many questions about the client's subjective experience, it also adds questions that take an inter-subjective approach (i.e., querying respondents 
about the perceived views of other people in their communities).

Other approaches to assessing shared models. In contexts where researchers know something about local concepts, but are not sure about whether the models that they know about are widely shared or coherent, other anthropological tools such as free listing, pile sorting, and consensus analysis can be used to examine people's understanding of a particular domain. These approaches are often grouped together because they can be used in sequence to move from open exploration to quantitative confirmation. They have grown increasingly popular in cognitive anthropology, including the study of health, but are less well-known in cultural psychology. In free listing, participants are asked to list words or short phrases (E. C. Thompson \& Juan, 2006). For example, the researcher might ask Punjabi participants to list the different names for mental illness or specific illnesses, the different ways one can tell that someone might be mentally ill, or the different treatment options. Once the words are generated, one must then sort the terms to eliminate redundancies and group together similar responses. In a cross-cultural study, this might involve making difficult decisions about linguistic equivalence across different languages.

In pile sorting, words or short phrases are compiled into a "deck of cards," either using a physical deck or computer software. In a sequential study, the stimuli on the cards might be derived from a previous free listing study by selecting those that were most commonly listed by different participants. Participants are then asked to sort the cards into at least two piles; there may or may not be constraints on the maximum number of piles (Bennardo \& Munck, 2014; Hines, 1993). In one variant, a hierarchical relation can be discerned by asking participants to take multiple passes, sorting the cards into a few large piles before breaking up each one into smaller piles. The results are amenable to various kinds of statistical analysis, such as multidimensional scaling, but can also be combined with a "think aloud" methodology in which participants are asked to talk through their thinking process as they sort the cards, with the resulting transcript analysed qualitatively (Bourey et al., 2012).

After generating relevant words and short phrases and then learning more about their structure, one can explore whether the commonly reported ones cohere as consensually understood models. Cultural consensus analysis provides a statistical approach to this (Dressler et al., 2015; Romney et al., 1986), allowing researchers to assess each participant's knowledge of the model and, hence, individual discrepancies. Newer tools such as symptom network analysis can be leveraged to assess beliefs about the interrelation of symptoms in a model (Schmittmann et al., 2013).

In addition, research on cultural shaping of psychological processes is moving toward data-driven methods that are used to examine similarities and differences in understandings of how lower level perceptual data (e.g., facial muscle movements) map onto the conceptual realm for concepts such as emotions (Jack et al., 2018). Similar methods can be used to understand the ways in which people conceptualise distress. This would require researchers to systematically generate large pools of stimuli offering different combinations of potential symptoms. Participants would be asked to judge such stimuli based on whether they map onto a known category or distress, thereby identifying combinations of symptoms that are consensually understood as meaningful in a given community.

\section{Where is the Local Consensual Boundary Between Nor- malcy and Deviancy?}

Another set of hypotheses that can be generated focusses on the impact of fuzzy conceptual boundaries between models of normalcy and deviancy. We have argued that the extent to which symptoms are conceptualised as normal-albeit potentially undesirable_-versus pathological has implications for intrapersonal and interpersonal impact of these symptoms. When comparing experiences of symptoms and patterns of observable behaviour across cultural contexts, it is important to consider how they are understood by both an individual person and by his or her community. To our knowledge, no existing cross-cultural clinical studies have systematically examined this question, although the fuzzy boundaries between pathological, problematic, and normal are known to be drawn differently in different contexts (e.g., work on psychotic experiences in the United States, India, and Ghana: T. M. Luhrmann et al. 2015). A researcher interested in this set of questions can utilise some of the tools for examining the ways in which people apply category thresholds when confronted with complex and noncategorical perceptual data. Such questions are important to the study of emotion and pain perception (Ludäscher et al., 2007; Pollak et al., 2000), social cognition (Hall \& Goh, 2017), clinical decisionmaking (C. Thompson et al., 2008), and risk assessment (Lerman et al., 2010), to mention just a few.

To understand how people draw boundaries in this domain, the researcher may create sets of written or filmed vignettes that vary continuously in their features (e.g., locally recognised manifestations of irritation systematically differing in intensity or duration of symptoms or interpersonal implications), asking local participants to judge them as optimal, normative, suboptimal, or indicative of a known illness. This would allow for estimates of consensual thresholds that differentiate, for example, models of normal and pathological irritability. Cross-cultural studies can compare such thresholds across contexts and examine whether personal or consensual understanding of certain symptoms as normative or pathological changes their impact. In cases where vignettes are based on actual cases, it may be possible to 
use self-related distress or impairment in a signal-detection paradigm, allowing closer examination of different types of response biases (e.g., false alarms, misses) in a given community.

\section{What Is the Relation Between Personal and Cultural Models and Between Models of Normalcy and Deviancy?}

The ability to capture people's personal model of normal and deviant functioning, along with their intersubjective understanding of the local cultural model, would allow researchers to examine the impact of discrepancies between these sets of models. The best set of examples of extant studies focussing on this set of questions comes from the work of Dressler et al. (2018); however, this work primarily examines discrepancies between personal and culturally normative models without examining models of deviancy. Research literatures on cultural consonance (Dressler, 2012), consensus analysis (Weller \& Mann, 1997), concept mapping (Trochim \& Kane, 2005), emotional acculturation (De Leersnyder et al., 2011), self-discrepancy (Higgins, 1987), and possible selves (Markus \& Nurius, 1986) all provide us with guidance on conceptualising and measuring the discrepancies between different models. Emerging approaches also show promise for large-scale semantic mapping (Cowen et al., 2019).

The ability to assess (a) how a given person and their community conceptualise a particular symptom, script, or cultural model and (b) the extent to which a given model of deviancy overlaps in content with salient models of normalcy would allow researchers to examine these models' implications for detection of, expression of, and responses to distress. Doing so could help generate within-culture and cross-cultural hypotheses that can be explored by clinicians and formally tested by researchers. For example, within Punjabi communities, do those with higher discrepancy between personal and normative models of emotional expression show a higher degree of impairment and less willingness to seek help? Do people in Punjabi-speaking communities conceptualise anger as more or less compatible with local normative models than do those in nearby Hindi-speaking or English-speaking communities? Improving our ability to conceptualise and address these issues is an important direction for future research.

Figure 2 Summary of key points for researchers and clinicians.

\section{Conclusion}

Think back to the case of Sukhbir Anand. Does the cultural models approach help us to better understand his own beliefs about his suffering, along with the beliefs of those around him? It can prompt us to think critically about the normalcy or deviancy, the valorisation and the devalorisation. It may even encourage future research on people living in similar contexts and suffering from similar problems, which in turn could improve clinical interventions. A brief summary of key points from this article, with implications for research and treatment, is shown in Figure 2. Two critical questions remain: (a) Can we use the cultural models approach to think differently about psychopathology, and (b) can we use it to improve the help that we can provide to specific clients? We therefore conclude by briefly considering these issues, pointing the reader to sources that examine them in more detail.

The cultural models approach can be used to demonstrate that different groups of people can hold different beliefs about the same psychological "phenomenon," be it normal (e.g., public interaction) or deviant (e.g., problematic anger). For much of this discussion, however, we have not emphasised that the "phenomenon itself" is changed by the model in which it is understood. The clinical literature has provided numerous examples of how beliefs about the implications of certain experiences, such as a racing heart or another's suddenly averted gaze, can trigger a cascade of physiological, emotional, and interpersonal consequences-consequences which can loop back and further shape the experience and expression of a set of symptoms (Chentova-Dutton \& Ryder, 2019; Kirmayer \& Sartorius, 2007; Ryder \& ChentsovaDutton, 2015). Perhaps Mr. Anand gets irritable, sees confusion in a customer's face, imagines his mother shaking her head with disappointment, and at some level if knows what they must be thinking - and grows angrier.

If cultural models are capable of shaping not just understandings of mental disorder but also the presentation of symptoms, evaluation and intervention become much more complicated (Ryder \& Chentsova-Dutton, 2015). A promising diagnostic innovation in recent years is the development of a means for clinicians to evaluate a client's personally and intersubjectively held beliefs about their suffering in context, such that diagnostic decisions can be informed by relevant cultural models (Lewis-Ferńandez et al., 2013). Such tools might help avoid diagnostic error and, moreover, directly improve treatment outcomes. Indeed, there is meta-analytic evidence that cross-cultural treatment is enhanced when client and clinician share a perspective on the suffering being treated (Benish et al., 2011). Where there are differences in fit between a client's and a clinician's models, the clinician needs to nonetheless build trust and move toward a consensual model.

Returning to the case of Mr. Anand, during the early stages of his treatment it appears that client and clinician are recruiting different models of both normalcy and deviancy to frame his presenting complaints (also see Kleinman 1978). The client's parents may indeed be working from yet another model. A cultural models approach can help us better address these issues by guiding both research and clinical practice. The approach may also encourage researchers and clinicians to think in more nuanced, and more helpful, ways about the 


\section{Figure 2}

Summary of key points for researchers and clinicians.

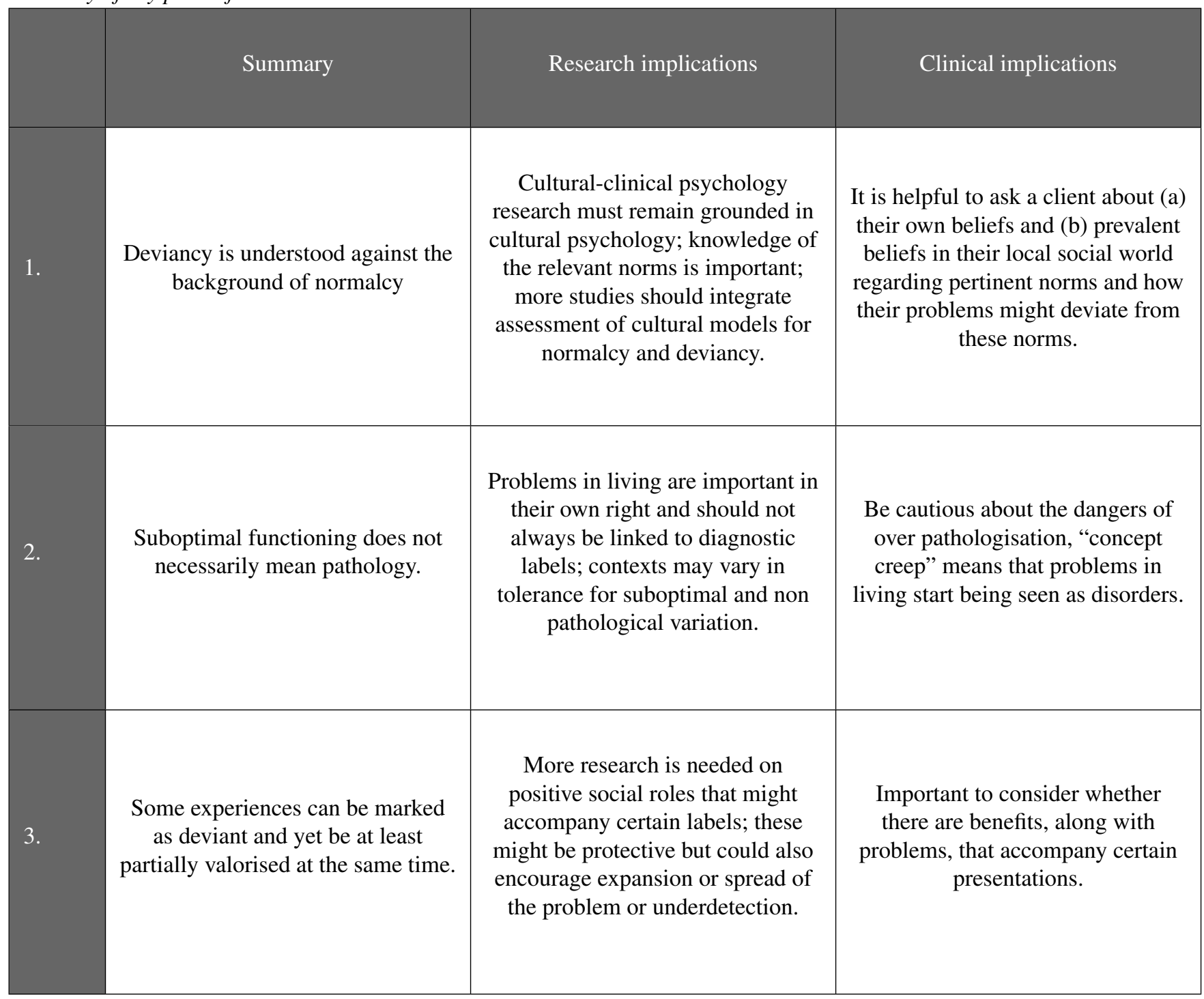

relation between the individual person and his or her context. The domain of culture and mental health remains largely uncharted (Chentova-Dutton \& Ryder, 2019). We hope the tools offered here will help researchers and clinicians who are setting out to map it.

\section{Conflict of Interest}

The authors have no conflict of interest to report.

\section{References}

Abdullah, T., \& Brown, T. L. (2011). Mental illness stigma and ethnocultural beliefs, values, and norms: An integrative review. Clinical Psychology Review, 31(6), 934-948.
Abelson, R. P. (1981). Psychological status of the script concept. American Psychologist, 36(7), 715.

American Psychiatric Association. (2013). Diagnostic and statistical manual of mental disorders, fifth edition (dsm-5). American Psychiatric Publishing.

Arcia, E., \& Fernandez, M. C. (1998). Cuban mothers' schemas of adhd: Development, characteristics, and help seeking behavior. Journal of Child and Family Studies, 7(3), 333-352.

Asnaani, A., Richey, J. A., Dimaite, R., Hinton, D. E., \& Hofmann, S. G. (2010). A cross-ethnic comparison of lifetime prevalence rates of anxiety disorders. The Journal of Nervous and Mental Disease, 198(8), 551. 
Bartholomew, R. E., Wessely, S., \& Rubin, G. J. (2012). Mass psychogenic illness and the social network: Is it changing the pattern of outbreaks? Journal of the Royal Society of Medicine, 105(12), 509-512.

Bateson, G. (1972). Steps toan ecology of mind. New York: Ballantine.

Benish, S. G., Quintana, S., \& Wampold, B. E. (2011). Culturally adapted psychotherapy and the legitimacy of myth: A direct-comparison meta-analysis. Journal of Counseling Psychology, 58(3), 279.

Bennardo, G., \& Kronenfeld, D. B. (2011). Types of collective representations: Cognition, mental architecture, and cultural knowledge. A companion to Cognitive Anthropology, 82-101.

Bennardo, G., \& Munck, V. C. d. (2014). Cultural models: Genesis, methods, and experiences. Oxford: Oxford University Press.

Bhui, K., \& Bhugra, D. (2002). Explanatory models for mental distress: Implications for clinical practice and research. The British Journal of Psychiatry, 181(1), 6-7.

Bourey, C., Stephenson, R., Bartel, D., \& Rubardt, M. (2012). Pile sorting innovations: Exploring gender norms, power and equity in sub-saharan africa. Global Public Health, 7(9), 995-1008.

Bruhn, J. G., Brandt Jr, E. N., \& Shackelford, M. (1966). Incidence of treated mental illness in three pennsylvania communities. American Journal of Public Health and the Nations Health, 56(6), 871-883.

Bruner, J. S. (1990). Acts of meaning (Vol. 3). Harvard university press.

Cafri, G., Yamamiya, Y., Brannick, M., \& Thompson, J. K. (2005). The influence of sociocultural factors on body image: A meta-analysis. Clinical Psychology: Science and Practice, 12(4), 421-433.

Cai, H., Kwan, V. S., \& Sedikides, C. (2012). A sociocultural approach to narcissism: The case of modern china. European Journal of Personality, 26(5), 529-535.

Carragher, N., Krueger, R. F., Eaton, N. R., \& Slade, T. (2015). Disorders without borders: Current and future directions in the meta-structure of mental disorders. Social Psychiatry and Psychiatric Epidemiology, 50(3), 339-350.

Chen, X., Wang, L., \& Cao, R. (2011). Shyness-sensitivity and unsociability in rural chinese children: Relations with social, school, and psychological adjustment. Child Development, 82(5), 1531-1543.

Chen, X., Wang, L., \& Wang, Z. (2009). Shyness-sensitivity and social, school, and psychological adjustment in rural migrant and urban children in china. Child Development, 80(5), 1499-1513.

Chentova-Dutton, Y. E., \& Ryder, A. G. (2019). Culturalclinical psychology. In D. Cohen \& S. Kitayama
(Eds.), Handbook of cultural psychology (2nd ed., pp. 365-396). Guilford Press.

Chirkov, V. (2016). Fundamentals of research on culture and psychology: Theory and methods. Routledge.

Chisuwa, N., \& O'Dea, J. A. (2010). Body image and eating disorders amongst japanese adolescents. a review of the literature. Appetite, 54(1), 5-15.

Chiu, C.-Y., Gelfand, M. J., Yamagishi, T., Shteynberg, G., \& Wan, C. (2010). Intersubjective culture: The role of intersubjective perceptions in cross-cultural research. Perspectives on Psychological Science, 5(4), 482-493.

Clark, H. H., \& Brennan, S. E. (1991). Grounding in communication.

Cline, R. J., \& Haynes, K. M. (2001). Consumer health information seeking on the internet: The state of the art. Health Education Research, 16(6), 671-692.

Coles, M. E., \& Coleman, S. L. (2010). Barriers to treatment seeking for anxiety disorders: Initial data on the role of mental health literacy. Depression and Anxiety, 27(1), 63-71.

Cooke, D. J., \& Michie, C. (1999). Psychopathy across cultures: North america and scotland compared. Journal of Abnormal Psychology, 108(1), 58.

Cooke, D. J., Michie, C., Hart, S. D., \& Clark, D. (2005). Assessing psychopathy in the uk: Concerns about cross-cultural generalisability. The British Journal of Psychiatry, 186(4), 335-341.

Cowen, A., Sauter, D., Tracy, J. L., \& Keltner, D. (2019). Mapping the passions: Toward a high-dimensional taxonomy of emotional experience and expression. Psychological Science in the Public Interest, 20(1), 69-90.

Crozier, I. (2011). Making up koro: Multiplicity, psychiatry, culture, and penis-shrinking anxieties. Journal of the History of Medicine and Allied Sciences, 67(1), 36-70.

Cruz, E. V., \& Higginbottom, G. (2013). The use of focused ethnography in nursing research. Nurse Researcher, 20(4).

Daley, T. C. (2004). From symptom recognition to diagnosis: Children with autism in urban india. Social Science $\mathcal{E}$ Medicine, 58(7), 1323-1335.

d'Andrade, R. G. (1995). The development of cognitive anthropology. Cambridge University Press.

De Leersnyder, J., Mesquita, B., \& Kim, H. S. (2011). Where do my emotions belong? a study of immigrants' emotional acculturation. Personality and Social Psychology Bulletin, 37(4), 451-463.

Ding, X., Coplan, R. J., Sang, B., Liu, J., Pan, T., \& Cheng, C. (2015). Young c hinese children's beliefs about the implications of subtypes of social withdrawal: 
A first look at social avoidance. British Journal of Developmental Psychology, 33(2), 159-173.

Draguns, J. G. (1980). Psychological disorders of clinical severity. In J. C. Draguns \& H. C. Triandis (Eds.), Handbook of cross-cultural psychology (pp. 99174). Allyn Bacon.

Dressler, W. W. (2004). Culture and the risk of disease. British Medical Bulletin, 69(1), 21-31.

Dressler, W. W. (2012). Cultural consonance: Linking culture, the individual and health. Preventive Medicine, 55(5), 390.

Dressler, W. W. (2017). Culture and the individual: Theory and method of cultural consonance. Routledge.

Dressler, W. W., Balieiro, M. C., \& Dos Santos, J. E. (2015). Finding culture change in the second factor: Stability and change in cultural consensus and residual agreement. Field Methods, 27(1), 22-38.

Dressler, W. W., Balieiro, M. C., \& dos Santos, J. E. (2018). What you know, what you do, and how you feel: Cultural competence, cultural consonance, and psychological distress. Frontiers in Psychology, 8, 2355.

Dumit, J. (2006). Illnesses you have to fight to get: Facts as forces in uncertain, emergent illnesses. Social Science $\mathcal{E}$ Medicine, 62(3), 577-590.

Ford, B. Q., Mauss, I. B., \& Gruber, J. (2015). Valuing happiness is associated with bipolar disorder. Emotion, 15(2), 211.

Foster, J. D., Campbell, W. K., \& Twenge, J. M. (2003). Individual differences in narcissism: Inflated self-views across the lifespan and around the world. Journal of Research in Personality, 37(6), 469-486.

Garcia, H. A., Finley, E. P., Lorber, W., \& Jakupcak, M. (2011). A preliminary study of the association between traditional masculine behavioral norms and ptsd symptoms in iraq and afghanistan veterans. Psychology of Men $\mathcal{E}$ Masculinity, 12(1), 55.

Gelfand, M. J., \& Harrington, J. R. (2015). The motivational force of descriptive norms: For whom and when are descriptive norms most predictive of behavior? Journal of Cross-Cultural Psychology, 46(10), 1273-1278.

Gelfand, M. J., Raver, J. L., Nishii, L., Leslie, L. M., Lun, J., Lim, B. C., Duan, L., Almaliach, A., Ang, S., Arnadottir, J., Et al. (2011). Differences between tight and loose cultures: A 33-nation study. Science, 332(6033), 1100-1104.

Giosan, C., Glovsky, V., \& Haslam, N. (2001). The lay concept of 'mental disorder': A cross-cultural study. Transcultural Psychiatry, 38(3), 317-332.

Gomes, M., Palmini, A., Barbirato, F., Rohde, L. A., \& Mattos, P. (2007). Knowledge about attention-deficit hyperactivity disorder in brazil. Jornal Brasileiro de Psiquiatria, 56(2), 94-101.

Groleau, D., Young, A., \& Kirmayer, L. J. (2006). The mcgill illness narrative interview (mini): An interview schedule to elicit meanings and modes of reasoning related to illness experience. Transcultural Psychiatry, 43(4), 671-691.

Hacking, I. (1992). Multiple personality disorder and its hosts. History of the Human Sciences, 5(2), 3-31.

Hall, J. A., \& Goh, J. X. (2017). Studying stereotype accuracy from an integrative social-personality perspective. Social and Personality Psychology Compass, 11(11), e12357.

Harnad, S. (2003). Categorical perception. In L. Nadel (Ed.), Encyclopedia of cognitive science (pp. 169-177). London, United Kingdom: Nature/Macmillan.

Haslam, N. (2005). Dimensions of folk psychiatry. Review of general psychology, 9(1), 35-47.

Haslam, N. (2016). Concept creep: Psychology's expanding concepts of harm and pathology. Psychological Inquiry, 27(1), 1-17.

Heinrichs, N., Rapee, R. M., Alden, L. A., Bögels, S., Hofmann, S. G., Oh, K. J., \& Sakano, Y. (2006). Cultural differences in perceived social norms and social anxiety. Behaviour Research and Therapy, 44(8), 1187-1197.

Higgins, E. T. (1987). Self-discrepancy: A theory relating self and affect. Psychological Review, 94(3), 319.

Hines, A. M. (1993). Linking qualitative and quantitative methods in cross-cultural survey research: Techniques from cognitive science. American Journal of Community Psychology, 21(6), 729-746.

Hirsh, J. B., Mar, R. A., \& Peterson, J. B. (2012). Psychological entropy: A framework for understanding uncertainty-related anxiety. Psychological Review, 119(2), 304.

Hollan, D. (1997). The relevance of person-centered ethnography to cross-cultural psychiatry. Transcultural Psychiatry, 34(2), 219-234.

Jack, R. E., Crivelli, C., \& Wheatley, T. (2018). Data-driven methods to diversify knowledge of human psychology. Trends in Cognitive Sciences, 22(1), 1-5.

James, S., Fernandes, M., Navara, G. S., Harris, S., \& Foster, D. (2009). Problemas de nervos: A multivocal symbol of distress for portuguese immigrants. Transcultural Psychiatry, 46(2), 285-299.

Johnson, N. (1957). The three faces of eve [film]. Augusta, GA: Twentieth-Century Fox.

Jones, N. A., Ross, H., Lynam, T., Perez, P., \& Leitch, A. (2011). Mental models: An interdisciplinary synthesis of theory and methods. Ecology and Society, 16(1). 
Karadaĝ, F., Oguzhanoglu, N. K., Özdel, O., Ateşci, F. Ç., \& Amuk, T. (2006). Ocd symptoms in a sample of turkish patients: A phenomenological picture. Depression and Anxiety, 23(3), 145-152.

Karasz, A. (2005). Cultural differences in conceptual models of depression. Social Science $\mathcal{E}$ Medicine, 60(7), 1625-1635.

Keller, J. D. (1992). Schemes for schemata. In T. Schwartz, G. M. White, \& C. A. Lutz (Eds.), New directions in psychological anthropology (pp. 59-67). Cambridge, United Kingdom: Cambridge University Press.

Kim, N. S., \& Ahn, W.-k. (2002a). Clinical psychologists' theory-based representations of mental disorders predict their diagnostic reasoning and memory. Journal of Experimental Psychology: General, 131(4), 451.

Kim, N. S., \& Ahn, W.-k. (2002b). The influence of naive causal theories on lay concepts of mental illness. American Journal of Psychology, 115(1), 33-66.

Kirmayer, L. J. (2005). Culture, context and experience in psychiatric diagnosis. Psychopathology, 38(4), 192-196.

Kirmayer, L. J. (2006). Beyond the 'new cross-cultural psychiatry': Cultural biology, discursive psychology and the ironies of globalization. Transcultural Psychiatry, 43(1), 126-144.

Kirmayer, L. J., \& Sartorius, N. (2007). Cultural models and somatic syndromes. Psychosomatic Medicine, 69(9), 832-840.

Kleinman, A. (1974). Cognitive structure of traditional medical. Ethnomedicine, 3, 27-49.

Kleinman, A. (1977). Depression, somatization and the "new cross-cultural psychiatry". Social Science $\mathcal{E}$ Medicine (1967), 11(1), 3-9.

Kleinman, A. (1978). Concepts and a model for the comparison of medical systems as cultural systems. Social Science $\mathcal{E}$ Medicine. Part B: Medical Anthropology, 12, 85-93.

Kohrt, B. A., \& Hruschka, D. J. (2010). Nepali concepts of psychological trauma: The role of idioms of distress, ethnopsychology and ethnophysiology in alleviating suffering and preventing stigma. Culture, Medicine, and Psychiatry, 34(2), 322-352.

Kotov, R., Krueger, R. F., Watson, D., Achenbach, T. M., Althoff, R. R., Bagby, R. M., Brown, T. A., Carpenter, W. T., Caspi, A., Clark, L. A., Et al. (2017). The hierarchical taxonomy of psychopathology (hitop): A dimensional alternative to traditional nosologies. Journal of Abnormal Psychology, 126(4), 454.

Kronenfeld, D. B. (2008). Culture, society, and cognition: Collective goals, values, action, and knowledge (Vol. 3). Walter de Gruyter.
Kronenfeld, D. B. (2011). One cognitive view of culture. A Companion to Cognitive Anthropology, 569-583.

Krueger, R. F., Chentsova-Dutton, Y. E., Markon, K. E., Goldberg, D., \& Ormel, J. (2003). A cross-cultural study of the structure of comorbidity among common psychopathological syndromes in the general health care setting. Journal of Abnormal Psychology, 112(3), 437.

Lerman, D. C., Tetreault, A., Hovanetz, A., Bellaci, E., Miller, J., Karp, H., Mahmood, A., Strobel, M., Mullen, S., Keyl, A., Et al. (2010). Applying signaldetection theory to the study of observer accuracy and bias in behavioral assessment. Journal of Applied Behavior Analysis, 43(2), 195-213.

LeVine, R. A. (1982). Culture, behavior, and personality. Transaction Publishers.

Lewis-Ferńandez, R., Aggarwal, N., Hinton, L., Hinton, D., Kirmayer, L. J., Et al. (2013). Dsm-5® handbook on the cultural formulation interview. American Psychiatric Pub.

Littlewood, R. (1990). From categories to contexts: A decade of the 'new cross-cultural psychiatry'. The British Journal of Psychiatry, 156(3), 308-327.

Liu, J., Chen, X., Coplan, R. J., Ding, X., Zarbatany, L., \& Ellis, W. (2015). Shyness and unsociability and their relations with adjustment in chinese and canadian children. Journal of Cross-Cultural Psychology, 46(3), 371-386.

Liu, J., Coplan, R. J., Chen, X., Li, D., Ding, X., \& Zhou, Y. (2014). Unsociability and shyness in c hinese children: Concurrent and predictive relations with indices of adjustment. Social Development, 23(1), 119-136.

Lloyd, K. R., Jacob, K. S., Patel, V., St. Louis, L., Bhugra, D., Mann, A., Et al. (1998). The development of the short explanatory model interview (semi) and its use among primary-care attenders with common mental disorders. Psychological Medicine, 28(5), 1231-1237.

Loo, P.-W., Wong, S., \& Furnham, A. (2012). Mental health literacy: A cross-cultural study from britain, hong kong and malaysia. Asia-Pacific Psychiatry, 4(2), 113-125.

Ludäscher, P., Bohus, M., Lieb, K., Philipsen, A., Jochims, A., \& Schmahl, C. (2007). Elevated pain thresholds correlate with dissociation and aversive arousal in patients with borderline personality disorder. Psychiatry Research, 149(1-3), 291-296.

Luhrmann. (2000). Of two minds: The growing disorder in american psychiatry. 2000. New York: Alfred A. Knopf.

Luhrmann, T. M., Padmavati, R., Tharoor, H., \& Osei, A. (2015). Differences in voice-hearing experiences of 
people with psychosis in the usa, india and ghana: Interview-based study. The British Journal of Psychiatry, 206(1), 41-44.

Markus, H., \& Nurius, P. (1986). Possible selves. American Psychologist, 41(9), 954.

Marsella, A. J., \& Yamada, A. M. (2007). Culture and psychopathology. Handbook of Cultural Psychology, 797-818.

Martin, E. (2009). Bipolar expeditions: Mania and depression in american culture. Princeton University Press.

Meylakhs, P., Rykov, Y., Koltsova, O., \& Koltsov, S. (2014). An aids-denialist online community on a russian social networking service: Patterns of interactions with newcomers and rhetorical strategies of persuasion. Journal of Medical Internet Research, 16(11), e261.

Michalak, L., \& Trocki, K. (2006). Alcohol and islam: An overview. Contemporary Drug Problems, 33(4), 523-562.

Minsky, M. (1975). A framework for representing knowledge. In P. Winston (Ed.), The psychology of computer vision (pp. 211-277). McGraw-Hill, New York.

Molloy, H., \& Vasil, L. (2004). Asperger syndrome, adolescence, and identity: Looking beyond the label. Jessica Kingsley Publishers.

Na, S., Ryder, A. G., \& Kirmayer, L. J. (2016). Toward a culturally responsive model of mental health literacy: Facilitating help-seeking among east asian immigrants to north america. American Journal of Community Psychology, 58(1-2), 211-225.

Napier, A. D., Ancarno, C., Butler, B., Calabrese, J., Chater, A., Chatterjee, H., Guesnet, F., Horne, R., Jacyna, S., Jadhav, S., Et al. (2014). Culture and health. The Lancet, 384(9954), 1607-1639.

Nations, M. K., Camino, L. A., \& Walker, F. B. (1988). nerves': Folk idiom for anxiety and depression? Social Science $\mathcal{E}$ Medicine, 26(12), 1245-1259.

Nichter, M. (2010). Idioms of distress revisited. Culture, Medicine, and Psychiatry, 34(2), 401-416.

Park, S., Lee, Y., Lee, E.-S., \& Kim, C. E. (2018). Public recognition of attention-deficit hyperactivity disorder in korea: Correct identification, causes, treatments, and social distance. Asian Journal of Psychiatry, 38, 3-8.

Pescosolido, B. A., Jensen, P. S., Martin, J. K., Perry, B. L., Olafsdottir, S., \& Fettes, D. (2008). Public knowledge and assessment of child mental health problems: Findings from the national stigma studychildren. Journal of the American Academy of Child $\mathcal{E}$ Adolescent Psychiatry, 47(3), 339-349.

Petrie, D. (1976). Sybil [film]. Lorimar Productions.
Pollak, S. D., Cicchetti, D., Hornung, K., \& Reed, A. (2000). Recognizing emotion in faces: Developmental effects of child abuse and neglect. Developmental Psychology, 36(5), 679.

Prentice, D. A., \& Miller, D. T. (2007). Psychological essentialism of human categories. Current Directions in Psychological Science, 16(4), 202-206.

Proulx, T., \& Inzlicht, M. (2012). The five "a" s of meaning maintenance: Finding meaning in the theories of sense-making. Psychological Inquiry, 23(4), 317335.

Punshon, C., Skirrow, P., \& Murphy, G. (2009). Thenot guilty verdict' psychological reactions to a diagnosis of asperger syndrome in adulthood. Autism, 13(3), 265-283.

Quinn, N. (2011). The history of the cultural models school reconsidered: A paradigm shift in cognitive. A companion to Cognitive Anthropology, 30.

Quinn, N., \& Holland, D. (1987). Culture and cognition. Cultural Models in Language and Thought, 3-40.

Rashid, M., Caine, V., \& Goez, H. (2015). The encounters and challenges of ethnography as a methodology in health research. International Journal of Qualitative Methods, 14(5), 1-16.

Regier, T., \& Kay, P. (2009). Language, thought, and color: Whorf was half right. Trends in Cognitive Sciences, 13(10), 439-446.

Reisbeck, C. K. (1985). Realistic language comprehension. In A. M. Aitkenhead \& J. M. Slacks (Eds.), Issues in cognitive modeling. London, United Kingdom: Erlbaum.

Romney, A. K., Weller, S. C., \& Batchelder, W. H. (1986). Culture as consensus: A theory of culture and informant accuracy. American Anthropologist, 88(2), 313-338.

Rosch, E. H. (1973). On the internal structure of perceptual and semantic categories, In Cognitive development and acquisition of language. Elsevier.

Rüdell, K., Bhui, K., \& Priebe, S. (2009). Concept, development and application of a new mixed method assessment of cultural variations in illness perceptions: Barts explanatory model inventory. Journal of Health Psychology, 14(2), 336-347.

Rumelhart, D. E. (1980). On evaluating story grammars. Cognitive Science, 4(3), 313-316.

Ryder, A. G., \& Chentsova-Dutton, Y. E. (2015). Culturalclinical psychology: From cultural scripts to contextualized treatments. In L. J. Kirmayer, R. Lemelson, \& C. A. Cummings (Eds.), Re-visioning psychiatry: Cultural phenomenology, critical neuroscience, and global mental health. New York, NY: Cambridge University Press. 
Ryder, A. G., Ban, L. M., \& Chentsova-Dutton, Y. E. (2011). Towards a cultural-clinical psychology. Social and Personality Psychology Compass, 5(12), 960-975.

Saint Arnault, D., \& Shimabukuro, S. (2012). The clinical ethnographic interview: A user-friendly guide to the cultural formulation of distress and help seeking. Transcultural Psychiatry, 49(2), 302-322.

Schank, R. C., \& Abelson, R. P. (1977). Scripts, plans, goals, and understanding: An inquiry into human knowledge structures. Hillsdale, NJ: Erlbaum.

Schmittmann, V. D., Cramer, A. O., Waldorp, L. J., Epskamp, S., Kievit, R. A., \& Borsboom, D. (2013). Deconstructing the construct: A network perspective on psychological phenomena. New Ideas in Psychology, 3l(1), 43-53.

Schoeneman, T. J., Segerstrom, S., Griffin, P., \& Gresham, D. (1993). The psychiatric nosology of everyday life: Categories in implicit abnormal psychology. Journal of Social and Clinical Psychology, 12(4), 429453.

Scholtens, A. (1991). Planning in ordinary conversation. Journal of Pragmatics, 16(1), 31-58.

Schreiber, F. R. (1973). Sybil. Chicago, IL: Henry Regnery.

Searle, J. R. (1980). The background of meaning, In Speech act theory and pragmatics. Springer.

Seligman, R. (2005). Distress, dissociation, and embodied experience: Reconsidering the pathways to mediumship and mental health. Ethos, 33(1), 71-99.

Seligman, R., \& Kirmayer, L. J. (2008). Dissociative experience and cultural neuroscience: Narrative, metaphor and mechanism. Culture, Medicine and Psychiatry, 32(1), 31-64.

Shore, B. (1998). Culture in mind: Cognition, culture, and the problem of meaning. Oxford University Press.

Siemer, M. (2008). Beyond prototypes and classical definitions: Evidence for a theory-based representation of emotion concepts. Cognition $\mathcal{E}$ Emotion, 22(4), 620-632.

Stadtmann, M. P., Maercker, A., Binder, J., \& Schnepp, W. (2018). Why do i have to suffer? symptom management, views and experiences of persons with a cptsd: A grounded theory approach. BMCpsychiatry, 18(1), 392.

Stice, E. (2001). Risk factors for eating pathology: Recent advances and future directions.

Tanaka-Matsumi, J., \& Draguns, J. G. (1997). Culture and psychopathology. In J. Berry, M. H. Segall, \& K. C (Eds.), Handbook of cross-cultural psychology (2nd ed., vol. 3): Social behavior and applications (pp. 449-492). Boston: Allyn \& Bacon.

Thigpen, C. H., \& Cleckley, H. M. (1954). A case of multiple personality. The Journal of Abnormal and Social Psychology, 49(1), 135.
Thigpen, C. H., \& Cleckley, H. M. (1957). The three faces of eve.

Thoits, P. A. (1985). Self-labeling processes in mental illness: The role of emotional deviance. American Journal of Sociology, 91(2), 221-249.

Thompson, C., Dalgleish, L., Bucknall, T., Estabrooks, C., Hutchinson, A. M., Fraser, K., de Vos, R., Binnekade, J., Barrett, G., \& Saunders, J. (2008). The effects of time pressure and experience on nurses' risk assessment decisions: A signal detection analysis. Nursing Research, 57(5), 302-311.

Thompson, E. C., \& Juan, Z. (2006). Comparative cultural salience: Measures using free-list data. Field Methods, 18(4), 398-412.

Trochim, W., \& Kane, M. (2005). Concept mapping: An introduction to structured conceptualization in health care. International Journal for Quality in Health Care, 17(3), 187-191.

Twamley, E. W., \& Davis, M. C. (1999). The sociocultural model of eating disturbance in young women: The effects of personal attributes and family environment. Journal of social and clinical psychology, 18(4), 467-489.

Wall, S. (2014). Focused ethnography: A methodological adaption for social research in emerging contexts.

Walsh, Z. (2013). Psychopathy and criminal violence: The moderating effect of ethnicity. Law and Human Behavior, 37(5), 303.

Ware, N. C. (1992). Suffering and the social construction of illness: The delegitimation of illness experience in chronic fatigue syndrome. Medical Anthropology Quarterly, 6(4), 347-361.

Wassmann, J., Kluge, C., \& Albrecht, D. (2011). The cognitive context of cognitive anthropology. A Companion to Cognitive Anthropology, 47-60.

Weiss, M. (1997). Explanatory model interview catalogue (emic): Framework for comparative study of illness. Transcultural Psychiatry, 34(2), 235-263.

Weller, S. C., \& Mann, N. C. (1997). Assessing rater performance without a" gold standard" using consensus theory. Medical Decision Making, 17(1), 71-79.

Wernke, M. R., \& Huss, M. T. (2008). An alternative explanation for cross-cultural differences in the expression of psychopathy. Aggression and Violent Behavior, 13(3), 229-236.

Williams, M. T., Wetterneck, C., Tellawi, G., \& Duque, G. (2015). Domains of distress among people with sexual orientation obsessions. Archives of Sexual Behavior, 44(3), 783-789.

Wisdom, J. P., \& Green, C. A. (2004). being in a funk": Teens' efforts to understand their depressive experiences. Qualitative Health Research, 14(9), 1227-1238. 
Witztum, E., Latzer, Y., \& Stein, D. (2008). Anorexia nervosa and bulimia nervosa as idioms of distress: From the historical background to current formulations. Int $J$ Child Adolesc Health, 1(4), 283-94.

Woodward, R. V., Broom, D. H., \& Legge, D. G. (1995). Diagnosis in chronic illness: Disabling or enablingthe case of chronic fatigue syndrome. Journal of the Royal Society of Medicine, 88(6), 325.

Yorulmaz, O., \& Iş1k, B. (2011). Cultural context, obsessivecompulsive disorder symptoms, and cognitions: A preliminary study of three turkish samples living in different countries. International Journal of Psychology, 46(2), 136-143.

Yunus, M., Masi, A. T., Calabro, J. J., Miller, K. A., \& Feigenbaum, S. L. (1981). Primary fibromyalgia (fibrositis): Clinical study of 50 patients with matched normal controls, In Seminars in arthritis and rheumatism. Elsevier.

Zohar, A. H., Goldman, E., Calamary, R., \& Mashiah, M. (2005). Religiosity and obsessive-compulsive behavior in israeli jews. Behaviour Research and Therapy, 43(7), 857-868. 